[0212-7199 (2005) 22: 4; pp 172-176] ANALES DE MEDICINA INTERNA Copyright (@) 2005 ARAN EDICIONES, S.L

AN. MED INTERNA (Madrid) Vol. 22, N. ${ }^{\circ} 4$, pp. 172-176, 2005

\title{
Perfil metabólico y deterioro de órganos diana en hipertensos esenciales según el control de la presión arterial en la consulta
}

\author{
S. GONZÁLEZ QUIJADA, M. ASTORGA ROMÓN'
}

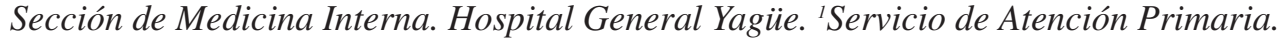
Burgos

METABOLIC PROFILE AND END-ORGAN DAMAGE IN ESSENTIAL HYPERTENSIVE PATIENTS ACCORDING TO THE CONTROL OF BLOOD PRESSURE IN PRIMARY CARE CENTERS

\section{RESUMEN}

Fundamento u objetivo: Analizar el grado de control metabólico y deterioro de los órganos diana en una muestra de hipertensos clasificados según el control de la presión arterial (PA) en consultas de atención primaria.

Pacientes y métodos: Estudio observacional trasversal de intervención diagnóstica realizado en 16 centros de atención primaria de la provincia de Burgos. Se reclutaron 207 hipertensos con tratamiento farmacológico (120 con buen control de la PA y 87 mal control). Se realizó historia clínica, determinación de PA casual, monitorización ambulatoria de la PA (MAPA), electrocardiograma, y analítica en sangre y orina de 24 horas.

Resultados: El 32 y $31 \%$ respectivamente seguían tratamiento farmacológico combinado. No se detectaron diferencias significativas en los niveles de HDL colesterol $>130 \mathrm{mg} / \mathrm{dl}(62 \%$ vs. $62 \%)$, microalbuminúria $(5 \%$ vs. $10 \%)$ y glucemia basal $>126 \mathrm{mg} / \mathrm{dl}(10 \%$ vs. $15 \%)$ entre los dos grupos. Se encontró una proporción significativamente mayor de hipertensos mal controlados con hipertrofia del ventrículo izquierdo (HVI) $(13 \%$ vs. 34\%; p < 0,001), deterioro de la función renal (3\% vs. $11 \% ; \mathrm{p}<0,05)$, valor hematocrito medio $(41,9 \pm 4,5$ vs. $43,7 \pm 3,5 ; \mathrm{p}<$ $0,01)$ y distinto grado de control según MAPA $(1,6 \%$ vs. $16 \%$ : $\mathrm{p}<0,01)$.

Conclusiones: Nuestros hipertensos con mal control de la PA en la consulta presentan mayor deterioro de los órganos diana que aquellos con buen control, a pesar de presentar un perfil metabólico similar. El acceso a la MAPA por parte de atención primaria podría modificar la actitud terapéutica en un subgrupo significativo de hipertensos mal controlados.

PALABRAS CLAVE: Hipertensión arterial. Monitorización ambulatoria de la presión arterial. Factores de riesgo cardiovascular.

\begin{abstract}
Background and objective: To evaluate the degree of metabolic control and end-organ damage in a group of hypertensive patients with poor and good blood pressure $(B P)$ control in primary health care centers.

Patients and methods: Observational study with diagnostic intervention, performed in 16 primary care centers of Burgos (Spain). 207 patients (120 with good BP control and 87 with poor BP control) with pharmacological treatment were included in the study. The following parameters were determined: clinic history, casual BP, 24-h ambulatory blood pressure monitoring (ABPM), electrocardiography, and clinic analysis in plasma and 24-hour urine.

Results: The $32 \%$ and $31 \%$ of patients respectively were receiving combined pharmacological treatment. No significant differences were detected between the two groups for the levels of $c-H D L>130 \mathrm{mg} / \mathrm{dl}$ (62\% vs. 62\%), microalbuminuria (5\% vs. 10\%) and basal glucose levels $>126 \mathrm{mg} / \mathrm{dl}$ (105 vs. 15\%). There was a significant proportion of patients with poor clinical BP control with left ventricular hypertrophy (13\% vs. 34\%; $p<0,001)$, impaired renal function (3\% vs. $11 \% ; p<$ $0,05)$, mean hematocrit value $(41,9 \pm 4,5$ vs. $43,7 \pm 3,5 ; p<0,01)$ and different degree on BP control by $A B P M(1,6 \%$ vs. $16 \%: p<0,01)$.

Conclusions: Our hypertensive patients with poor BP clinical control showed more end-organ damage than those with good control, alt hough they have a similar metabolic profile. The access to ABPM by primary health care centers could modify the therapeutic management in a significant subgroup of hypertensive patients with poor BP control.
\end{abstract}

KEY WORDS: Arterial hipertensión. Ambulatory blood pressure monitoring $(A B P M)$. Cardiovascular risk factor.

González Quijada S, Astorga Romón M. Perfil metabólico y deterioro de órganos diana en hipertensos esenciales según el control de la presión arterial en la consulta. An Med Interna (Madrid) 2005; 22: 172-176.

\section{INTRODUCCIÓN}

Uno de los objetivos prioritarios para reducir la mortalidad cardiovascular es conseguir un aceptable control de la hipertensión arterial (HTA), así como, el de otros factores de riesgo cardiovascular. Un porcentaje variable, pero importante, de hipertensos atendidos en atención primaria mantienen cifras tensionales elevadas. En el estudio PRESCAP realizado en 12.754 pacientes, se observó que sólo 4 de cada 10 hipertensos tratados farmacológicamente y atendidos en el ámbito de atención primaria de nuestro país tienen un control óptimo de la presión arterial (PA) (1). Los factores asociados al mal con- 
trol de la PA en este estudio fueron el consumo elevado de alcohol, el sedentarismo, la obesidad y la edad (1). Otros factores implicados de forma más directa han sido la falta de observancia del tratamiento, prescripción inadecuada o insuficiente y deficiente seguimiento del paciente (2). En otras ocasiones se trata de "falsos mal controlados", fenómeno asociado a la variabilidad propia de la PA y designada habitualmente como HTA clínica aislada (HCA) o de "bata blanca" (detección de cifras elevadas de PA exclusivamente en la consulta) (3).

Las decisiones terapéuticas en los sujetos hipertensos se basan también en el nivel de riesgo cardiovascular y el deterioro de órganos diana. Estas deben abarcar cambios en el estilo de vida y terapia farmacológica para conseguir una estratificación de riesgo lo más bajo posible. Sin embargo, el grado de control conseguido en conjunto es con frecuencia inadecuado $(1,3)$.

El objetivo de este estudio es analizar el grado de control metabólico y deterioro de los órganos diana en una muestra de hipertensos clasificados según el control de la PA en la consulta. Como objetivo secundario figura el impacto que podría suponer la monitorización ambulatoria de la PA (MAPA) en el manejo terapéutico de estos pacientes.

\section{MATERIAL Y MÉTODO}

Se trata de un estudio observacional realizado en hipertensos previamente diagnosticados y atendidos en 16 centros de salud (12 rurales y 4 urbanos) de atención primaria de la provincia de Burgos. El muestreo fue no probabilístico y en base a obtener grupos comparables de hipertensos bien y mal controlados (PA repetidamente menor o mayor de $140 / 90 \mathrm{mmHg}$ en consulta). Para el diagnóstico previo de HTA se siguieron las recomendaciones previas de la OMS (4). Se incluyeron hipertensos ya conocidos que recibían tratamiento farmacológico. Los pacientes eran remitidos por su médico de cabecera, realizando un único facultativo de atención primaria la historia clínica y MAPA. En cada paciente se obtuvo un electrocardiograma estándar, hemograma, determinación de iones en sangre y orina de $24 \mathrm{~h}$, bioquímica general, perfil lipídico y microalbuminúria en orina de 24 horas.

La MAPA se realizó con un equipo validado (SpaceLabs 90202), con determinaciones seriadas cada 20 y 30 minutos durante los periodos de vigilia ( 8 a 22 h) y sueño (22 a 8 h) respectivamente. Los parámetros analizados y criterios de utilización fueron los comúnmente establecidos. Se adoptaron como valores límites o normales de la MAPA los sugeridos por Pickering y cols. (PA media $<135 / 85 \mathrm{mmHg}$ en 24 horas) (5). Se definieron como no dipper aquellos sujetos con descensos nocturnos de la PAS y de la PAD inferiores al $10 \%$ de la PA en el periodo de día (6). La carga tensional se estimó como el porcentaje de lecturas superiores a $140 / 90 \mathrm{mmHg}$ para el periodo de vigilia y a $120 / 80 \mathrm{mmHg}$ para el periodo de sueño.

El deterioro de órganos diana y el perfil metabólico se analizaron según las nuevas directrices de la Sociedad Europea de Hipertensión (3). La hipertrofia del ventrículo izquierdo (HVI) se determinó según el índice de SoKolow-Lyons (SV1 + RV5-6 > 38 mm; Cornell >2440 mm*ms). El deterioro de la función renal se determinó como el incremento de la creatinina sérica por encima de $1,4 \mathrm{mg} / \mathrm{dL}$ en el hombre y 1,5 $\mathrm{mg} / \mathrm{dL}$ en la mujer o un aclaramiento de creatinina inferior a $60 \mathrm{ml} / \mathrm{min}$ (3). La microalbuminúria se definió como la presencia de entre 30 y $300 \mathrm{mg}$ de albúmina en orina de 24 h (3). Se consideraron inadecuados la glucemia en ayunas superior a $126 \mathrm{mg} / \mathrm{dL}$. y el colesterol LDL (c-LDL) mayor de 130 $\mathrm{mg} / \mathrm{dL}$. Se entendió que el paciente seguía una actividad física recomendada cuando realizaba regularmente ejercicio moderado aeróbico, como caminar, correr o nadar, durante 30-45 minutos, tres o cuatro veces por semana (3).

Los datos continuos han sido expresados mediante la media y la desviación estándar (DE), y los dicotómicos en frecuencias relativas o porcentajes. En el caso de las variables continuas se realizó la comprobación de ajuste a una distribución normal aplicando la prueba de Kolmogorov-Smirnov. El test de la t de Student se empleó para comparar entre subgrupos (hipertensos controlados y no controlados) los valores medios de las variables continuas a estudio y el $\chi^{2}$ para los porcentajes. En todos los casos se consideró el test realizado como estadísticamente significativo cuando $\mathrm{p}$ era menor de 0,05, es decir, para un nivel de confianza del $95 \%$.

\section{RESULTADOS}

Las características de los pacientes en función del criterio de buen o mal control de la PA en la consulta clínica se muestran en la tabla I. Se incluyeron 207 hipertensos, de los cuales mostraban buen control de la PA 120 y mal control 87. La edad media fue de 62 y 64 años respectivamente y la mayoría de los pacientes presentaban una HTA leve-moderada (93\% y 99\%). El 95\% reunían dos o más factores de riesgo cardiovascular. Los hábitos de vida, control metabólico y deterioro de los órganos diana de los pacientes según el control de la PA se muestran en la tabla II. Los dos grupos resultaron equipara-

\section{TABLA I}

CARACTERÍSTICAS DE LOS PACIENTES EN FUNCIÓN DEL CRITERIO DE BUEN O MAL CONTROL DE LA PA EN CONSULTA

\begin{tabular}{lcc}
\hline & $\begin{array}{c}\text { Buen control } \\
(n=120)\end{array}$ & $\begin{array}{c}\text { Control deficiente } \\
(n=87)\end{array}$ \\
\hline Edad (años) & $62 \pm 10$ & $64 \pm 12$ \\
Varones & $44(37 \%)$ & $42(48 \%)$ \\
IMC $\left(\mathrm{kg} / \mathrm{m}^{2}\right)$ & $29 \pm 4$ & $29 \pm 4$ \\
Hipertensión leve/moderada & $111(93 \%)$ & $86(99 \%)$ \\
Hipertensión < 10 años de evolución & $50(42 \%)$ & $35(40 \%)$ \\
Antecedentes de eventos cardiovasculares & $6(5 \%)$ & $6(7 \%)$ \\
Diabetes mellitus & $9(8 \%)$ & $7(8 \%)$ \\
Tratamiento hipolipemiante & $23(19 \%)$ & $12(14 \%)$ \\
PAS media de 24 h & $120 \pm 8$ & $141 \pm 10 \mathrm{a}$ \\
PAD media de 24 h & $72 \pm 6$ & $80 \pm 8 \mathrm{a}$ \\
Carga sistólica de la PA & $18,2 \pm 14,1$ & $64,0 \pm 21,8^{\mathrm{a}}$ \\
Carga diastólica de la PA & $8,9 \pm 8,9$ & $32,2 \pm 25,4^{\mathrm{a}}$ \\
Dippers & $84(70 \%)$ & $62(71 \%)$ \\
Posible cambio de actitud según MAPA & $2(1,6 \%)$ & $14(16 \%)^{\mathrm{b}}$ \\
\hline Resultados expros
\end{tabular}

Resultados expresados en número total de pacientes (\%) o media \pm desviación estándar; ${ }^{a}: p<0,001 ;{ }^{b}$ : Pacientes en los que al realizar monitorización ambulatoria de la PA cambiarían supuestamente de subgrupo (buen a mal control y viceversa) según una PA media $<135 / 85 \mathrm{mmHg}$ en 24 horas; PAS: presión arterial sistólica en $\mathrm{mmHg}$; PAD: presión arterial diastólica en $\mathrm{mmHg}$. 
TABLA II

HÁBITOS DE VIDA, CONTROL METABÓLICO Y DETERIORO DE LOS ÓRGANOS DIANA DE LOS PACIENTES SEGÚN EL CONTROL DE LA PA

\begin{tabular}{lcc}
\hline & $\begin{array}{c}\text { Buen control } \\
(n=120)\end{array}$ & $\begin{array}{c}\text { Control deficiente } \\
(n=87)\end{array}$ \\
\hline IMC $>30$ & $52(43 \%)$ & $40(46 \%)$ \\
Fumadores activos & $11(9 \%)$ & $14(16 \%)$ \\
Ingesta mayor de 40 g etanol/día & $34(28 \%)$ & $30(34 \%)$ \\
Ejercicio aeróbico habitual & $2(2 \%)$ & $9(10 \%)^{\mathrm{a}}$ \\
Sodio en orina de $24 \mathrm{~h}(\mathrm{mmol} / \mathrm{L})$ & $129 \pm 58$ & $118 \pm 69$ \\
Clucemia $>126 \mathrm{mg} / \mathrm{dL}(>6,9 \mathrm{mmol} / \mathrm{L})$ & $12(10 \%)$ & $13(15 \%)$ \\
Ácido úrico $>7 \mathrm{mg} / \mathrm{dL}(>416,36$ umol/L) & $32(27 \%)$ & $25(29 \%)$ \\
Hematocrito $(\%)$ & $41,9 \pm 4,5$ & $43,7 \pm 3,5^{b}$ \\
Colesterol total $>250 \mathrm{mg} / \mathrm{dL}(>6,46 \mathrm{mmol} / \mathrm{L})$ & $17(14 \%)$ & $18(20 \%)$ \\
LDL colesterol > $130 \mathrm{mg} / \mathrm{dl}(>3,36 \mathrm{mmol} / \mathrm{L})$ & $69(62 \%)$ & $50(62 \%)$ \\
Microalbuminuria $(30-300 \mathrm{mg} / 24 \mathrm{~h})$ & $6(5 \%)$ & $9(10 \%)$ \\
Deterioro de la función renal & $4(3 \%)$ & $10(11 \%)^{\mathrm{a}}$ \\
Hipertrofia del VI (por EKC) & $15(13 \%)$ & $30(34 \%)^{\mathrm{c}}$ \\
\hline
\end{tabular}

Resultados expresados en número total de pacientes ( $\%$ ) o media \pm desviación estándar; IMC: índice de masa corporal; a: $\mathrm{p}<0,05$; b: $\mathrm{p}<0,01$; c: $p<0,001$; ${ }^{d}$ : Creatinina en sangre mayor de $1,5 \mathrm{mg} / \mathrm{dL}$ en hombres $y$ mayor de 1,4 mg/dL en mujeres; EKG: Electrocardiograma convencional de 12 derivaciones; VI: ventrículo izquierdo.

bles en cuanto a edad, sexo, tiempo de evolución de la hipertensión y hábitos tóxicos (tabaco y alcohol). El tratamiento farmacológico de los dos subgrupos de pacientes se muestra en la tabla III. Los resultados de la MAPA mostraban un mal control de la PA en el 1,6\% de los pacientes con buen control en la consulta y viceversa (PA media de $24 \mathrm{~h}<135 / 85$ ) en el $16 \%$ de aquellos con mal control $(\mathrm{p}<0,05)$.

Prácticamente la mitad de los pacientes eran obesos, sin diferencias significativas en la ingesta de sal reflejada por la excreción de sodio en orina de 24 h. Una proporción importante de pacientes en ambos grupos mantenían cifras inadecuadas de glucemia basal, c-LDL y ácido úrico (Tabla II). La proporción de pacientes con microalbuminuria en los hipertensos mal controlados era mayor, aunque este incremento no

TABLA III

TRATAMIENTO FARMACOLÓGICO DE LOS PACIENTES EN FUNCIÓN DEL CRITERIO DE BUEN O MAL CONTROL DE LA PA EN CONSULTA

\begin{tabular}{lcc}
\hline & $\begin{array}{c}\text { Buen control } \\
(n=120)\end{array}$ & $\begin{array}{c}\text { Control deficiente } \\
(n=87)\end{array}$ \\
\hline $\begin{array}{l}\text { Tratamiento farmacológico combinado } \\
\text { Diuréticos aislados o en combinación }\end{array}$ & $38(32 \%)$ & $27(31 \%)$ \\
Inhibidores de la enzima conversora de & $62(52 \%)$ & $40(46 \%)$ \\
$\quad$ la angiotensina & $50(42 \%)$ & $41(47 \%)$ \\
Antagonistas del receptor de la & $33(28 \%)$ & $25(29 \%)$ \\
$\quad$ angiotensina Il & $29(24 \%)$ & $29(33 \%)$ \\
Antagonistas del calcio & $9(11 \%)$ & $7(8 \%)$ \\
$\quad \beta$-Bloqueantes & $7(6 \%)$ & $9(10 \%)$ \\
$\alpha$-Bloqueantes &
\end{tabular}

Resultados expresados en número total de pacientes y $\%$. era significativo. Este subgrupo mostraba mayor proporción de sujetos con HVI, deterioro de la función renal y valor hematocrito (Tabla II). Este último se mantenía elevado independientemente de la ingesta de diuréticos y del hábito tabáquico. Al estratificar el valor hematocrito según el sexo, esta diferencia se mostraba solo significativa en las mujeres [varones hipertensos mal/bien controlados $(44,5 \pm 3,7$ vs. $45,3 \pm$ $3,2)$ y mujeres hipertensas $\mathrm{mal} / \mathrm{bien}$ controladas $(40,6 \pm 3,2$ vs. $42,5 \pm 2,7 ; \mathrm{p}<0,05)]$.

\section{DISCUSIÓN}

Se ha comprobado como cada factor de riesgo cardiovascular no controlado considerado individualmente es un importante causa de riesgo residual a pesar de un adecuado control de la PA (7). Por otro lado, es constante el hallazgo de una alta proporción de sujetos hipertensos con hábitos de vida inadecuados y que muestran un pobre control metabólico (2). En los pacientes hipertensos se recomienda el abandono del tabaco, reducción de peso, reducción de la ingesta de sal e ingesta excesiva de alcohol, ejercicio físico regular y seguir una dieta sana (dieta mediterránea). Sin embargo, no está demostrado que los cambios en el estilo de vida prevengan las complicaciones cardiovasculares en pacientes hipertensos (3).

La obesidad desempeña un papel importante en el desarrollo y mantenimiento de la HTA, de manera que su incidencia en obesos es de aproximadamente del $50 \%(1,8)$. En sentido inverso también es muy elevada la incidencia de obesidad en los hipertensos, tal como se demuestra en nuestra serie. Además del mayor riesgo de hipertensión, la obesidad se asocia con un aumento en los niveles de c-LDL, tolerancia a la glucosa disminuida y predisposición al desarrollo de HVI (7). En estos pacientes es más difícil el control de la hipertensión debido a que la obesidad puede interferir en la eficacia de los fármacos antihipertensivos. La pérdida de peso puede producir un descenso significativo de la PA, con lo que frecuentemente se puede reducir -e incluso prescindir- de la terapia antihipertensiva (9). Aunque los programas de intervención en este aspecto generalmente ofrecen escasos resultados a medio y largo plazo, no por ello se debe insistir en su importancia.

Es habitual que una proporción importante de pacientes hipertensos presentan cifras elevadas o inadecuadas de c-LDL (2). Numerosos estudios epidemiológicos han encontrado una asociación consistente entre los niveles de esta lipoproteina y la tasa de inciencia de enfermedad coronária (10). Un determinado nivel de colesterol sérico es un prerrequisito para la inducción de arteriosclerosis en modelos animales experimentales (11). Las LDL no sólo tienen un importante papel en el desarrollo de la placa en sus distintas fases, sino que también contribuye a su inestabilidad. En el estudio ASCOT se redujo el 36\% la incidencia de coronariopatía total y de infarto de miocardio no mortal, y el $27 \%$ la de accidentes crebrovasculares en más de 10.000 hipertensos tratados con $10 \mathrm{mg} /$ día de atorvastatina (12). El objetivo más comúnmente aceptado en los pacientes con dos o más factores de riesgo cardiovascular sería mantener el c-LDL por debajo de $130 \mathrm{mg} / \mathrm{dL}$ (13). Incluso esta cifra se esta reduciendo cada vez más en relación con las últimas evidencias (3). Observamos la discrepancia que existe entre estas recomendaciones y los resultados obtenidos, que se extiende a la mayoría de las series analizadas. 
La coexistencia de hipertensión y diabetes mellitus es frecuente y aumenta sustancialmente el riesgo de complicaciones cardiovasculares $(14,15)$. Con frecuencia estos pacientes muestran un "síndrome metabólico" donde coexisten además obesidad y dislipemia (16). Por otro lado, algunos fármacos, especialmente los diuréticos pueden inducir hiperglucemia en estos sujetos. Sin embargo, las consecuencias cardiovasculares de esta alteración metabólica inducida por fármacos a largo plazo no han sido bien establecida. Actualmente se ha comprobado que un tratamiento agresivo de la hipertensión (PA menor de 130/80 $\mathrm{mmHg}$ ) protege a los pacientes diabéticos de los acontecimientos cardiovasculares (17). Aunque nuestra proporción de hipertensos diabéticos es escasa, el número de aquellos con glucemias basales elevadas aumenta, quizás en relación con un efecto farmacológico.

Con frecuencia se observa hiperuricemia (definida como valores de urato sérico superiores a $7 \mathrm{mg} / \mathrm{dl}$ ) en pacientes hipertensos no tratados, habiéndose demostrado que estos valores se asocian con el desarrollo de nefroangioesclerosis (18). Franse y cols., en un amplio estudio prospectivo, encuentran que los niveles de ácido úrico predicen de forma independiente eventos cardiovasculares en personas de mayor edad con hipertensión sistólica aislada (19). En este estudio se subraya la necesidad de controlar los cambios en los niveles de ácido úrico durante el tratamiento diurético para ayudar a identificar aquellos pacientes que más se beneficiarían de una intervención terapéutica. Sin embargo, casi un tercio de nuestros pacientes permanecen con niveles descontrolados de ácido úrico. Parece prudente, por lo tanto, insistir en un mayor control de la hiperuricemia, especialmente en aquellos hipertensos que reciben diuréticos, de más edad o con deterioro de la función renal (19).

En algunos estudios epidemiológicos y en varias series descriptivas se ha observado una relación positiva entre los valores de PA y el hematocrito, tanto en hipertensos como en normotensos (20,21). Similares resultados obtenemos en nuestra serie, donde los hipertensos mal controlados exhiben un aumento significativo del valor hematocrito. Se ha descrito que la presencia de un hematocrito elevado es un factor independiente de riesgo para el desarrollo de ictus en sujetos en la segunda mitad de la vida (22). Posiblemente esta asociación esta en relación con el aumento de viscosidad que induce el aumento de la concentración de hematíes en la sangre, desconociendo en la actualidad cual es valor "seguro o normal" des- de el punto de vista cardiovascular. Los datos observados en nuestra serie apuntan a que un control adecuado de la PA puede inducir un descenso del hematocrito, y quizás, del riesgo cardiovascular sobreañadido. Como ya había descrito previamente nuestro grupo (21), parece que esta asociación es más evidente en el sexo femenino, sin poder precisar las causas de esta tendencia. Las consecuencias hemodinámicas derivadas de estos hallazgos se desconocen, pero habría que profundizar en sus posibles implicaciones y consecuencias.

Los hipertensos con mal control de la PA revisados en nuestra consulta presentan mayor deterioro de los órganos diana que aquellos con buen control, a pesar de presentar un perfil metabólico similar. Estos datos corroboran el importante papel de la PA como factor independiente y primordial de riesgo cardiovascular. Tanto la HVI como el deterioro de la función renal son potentes predictores independientes de morbomortalidad por causas cardiovasculares en hipertensos tratados $(7,23)$. La presencia de microalbuminúria en hipertensos no diabéticos es a la vez un marcador precoz de deterioro renal e indicador de aumento del riesgo cardiovascular (24). La comprobación de afectación orgánica en pacientes hipertensos exige una terapia más exigente, que permita detener, e incluso mejorar, los parámetros de deterioro orgánico subyacente (3). Por estos motivos parece inadecuado el control de la PA conseguido en una importante proporción de nuestros pacientes.

Recientemente se ha demostrado que la MAPA ofrece un valor pronóstico adicional en pacientes hipertensos con tratamiento, independientemente del grado de control de la PA en la consulta (25). Clement y cols., en un amplio estudio prospectivo, observan que la PA sistólica media ambulatoria de 24 horas mayor o igual de $135 \mathrm{mmHg}$ predice eventos cardiovasculares en hipertensos tratados, incluso después de su ajuste con la PA determinada en consulta y otros factores clásicos de riesgo cardiovascular (25). Por lo tanto, esta técnica podría ayudar a estratificar mejor el riesgo individual de los pacientes hipertensos y consecuentemente la adecuación del tratamiento. Siguiendo estos datos, hasta un $16 \%$ de nuestros pacientes mal controlados en la consulta se podrían beneficiar de un enfoque terapéutico más conservador, con repercusiones en gasto farmaceútico, prevención de efectos secundarios y adherencia al tratamiento. Sin embargo, queda por determinar la relación coste-beneficio que supondría su implantación generalizada a nivel de atención primaria.

\section{Bibliografía}

1. Llisterri JL, Rodríguez C, Alonso FJ, Lou S, División JA, Santos JA et al. Control de la presión arterial en la población hipertensa española atendida en atención primaria. Estudio PRESCAP 2002. Med Clin (Barc) 2004; 122: 165-71.

2. Hendrix KH, Lackland DT, Egan BM. Cardiovascular risk factor control and treatment patterns in primary care. Manag Care Interface 2003; 16: $21-26$

3. 2003 European Society of Hypertension-European Society of Cardiology; guidelines for the management of arterial hypertension. Guidelines Committee. Journal of Hypertension 2003; 21: 10011-1053.

4. 1999 WHO-ISH. Guidelines for the management of hypertensión. J Hipertens 1999; 17: 151-83.

5. Pickering TG, Gerin W, Schwartz AR. What is the white-coat effect and how should it be measured?. Blood Press Monit 2002; 7: 293-300.

6. Redón J, Vicente A, Álvarez A, Cremades B, Torró I, Tacons J, Lurbe
E. Variabilidad circadiana de la presión arterial: aspectos metodológicos para su estimación. Med Clin (Barc) 1999; 112: 285-289.

7. Zanchetti A, Hansson L, Dahlöf B, Elmfeldt D, Kjeldsen S, Kolloch R, et al. Effects of individual risk factors on the incidence of cardiovascular event in the treated hypertensive patients of the Hypertension Optimal Treattment Study. HOT Study Group. J Hypertens 2001; 19: 114959.

8. Wilson PW, D’Agostino RB, Sullivan L, Parise H, Kannel W. Overweigh and obesity as determinants of cardiovascular risk: the Framingham experience. Arch Intern Med 2002; 162: 1867-72.

9. Blumenthal JA, Sherwood A, Gullete EC, Babyak M, Waug R, Georgiades $\mathrm{A}$, et al. Exercice and weigh loss reduce blood pressure in men and woman with mild hypertension: Effects on cardiovascular, metabolic, and hemodynamic functioning. Arch Intern Med 2000; 160: $1947-$ 52. 
10. Wilson PW, D’Agostino RB, Levy D, Belanger AM, Silbershatz H, Kannel WB. Prediction of coronary heart disease using risk factor categories. Circulation 1998; 97: 1837-47.

11. Clair RW. The contribution of avian models to our understanding of atherosclerosis and their promise for the future. Lab Anim Sci 1998; 48: 565-68.

12. Sever PS, Dahlöf B, Pouler NR, Wedel H, Beevers G, Caulfield M, et al. for de ASCOT investigators. The prevention of coronary events and stroke with atorvastatin in hypertensive subjects with average or below average cholesterol levels. The Anglo-Scandinavian Cardiac Outcomes Trial: Lipid Lowering Arm (ASCOT: LLA). Lancet 2003; 361: 11491158.

13. Third report of the National Cholestrol Education Program (NCEP) Expert Panel on detection, evaluation, and treatment of high blood cholestrol in adults (Adult Treatment Panel III). Circulation 2002; 106: 3143-54.

14. Tarnow L, Rossing P, Gall MA, Nielsen FS, Parving HH. Prevalence of arterial hypertension in diabetic patients before and after the JNC-V. Diabetes Care 1994; 17: 1247-1251.

15. Hypertension in Diabetes Study (HDS): I. Prevalence of hypertension in newly presenting type 2 diabetic patients and the association with risk factors for cardiovascular and diabetic complications. J Hypertens 1993; 11: 309-17.

16. Reaven G. Metabolic syndrome: pathophysiology and inmplications for management of cardiovascular disease. Circulation 2002; 106: 286-288.

17. Dillon JJ. The quantitative relationship between treated blood pressure and progression of diabetic renal disease. Am J Kidney Dis 1993; 22: 798-802.
18. Messerli FH, Frohlich ED, Dreslinski GR, Suárez DH, Aristimuno GG. Serum uric acid in essential hypertension: an indicator of renal vascular involvement. Ann Intern Med 1980; 93: 817-21.

19. Franse LV, Pahor M, Di Bari M, Shorr RI, Wan JY, Somes GW, Applegate WB. Serum uric acid, diuretic treatment and risk of cardiovascular events in the systolic Hypertension in the Elderly Program (SHEP). J Hypertens 2000; 18: 1149-54.

20. Cirillo M, Laurenzi M, Trevisan M, Stamler J. Hematocrit, blood pressure and 21. González Quijada S, Luque Otero M, Fernández-Cruz A Almería Valera C. Asociación del hematocrito con la presión arterial y la masa ventricular izquierda en sujetos mayores de 55 años. Rev Clín Esp 1999; 199: 202-07.

21. González Quijada S, Luque Otero M, Fernández-Cruz A, Almería Valera C. Asociación del hematocrito con la presión arterial y la masa ventricular izquierda en sujetos mayores de 55 años. Rev Clín Esp 1999; 199: 202-07.

22. Wannamethee G, Perry IJ, Shaper AG. Haematocrit, hypertension and risk of stroke. J Intern Med 1994; 235: 163-68.

23. Levy D, Garrison RJ, Savage DB, Kanel WB, Castelli WP. Prognostic implications of echocardiographically determined left ventricular mass in the Framingham Heart Study. N Engl J Med 1990; 320: 1561-66.

24. Gerstein HC, Mann JF, Yi Q, Zinman B, Dinnen SF, Hoogwerf B et al. Albuminuria and risk of cardiovascular events, death, and heart failure in diabetic and nondiabetic individuals. JAMA 2001; 286: 421-26.

25. Clement DL, De Buyzere ML, De Bacquer DA, de Leeuw PW, Duprez DA, Fagard RH et al. Prognostic value of Ambulatory Blood - Pressure Recordings in patients with treated hypertension. N Engl J Med 2003; 348: 2377-78. 